\title{
Aarno Práticas sexuais e cuidados em saúde de mulheres que fazem sexo com mulheres: 2013-2014*
}

doi: $10.5123 /$ S1679-49742018000400005

\section{Sexual practices and health care of women who have sex with women:2013-2014}

Prácticas sexuales y cuidados en salud de mujeres que tienen sexo con mujeres: 2013-2014

\author{
Andréa Cronemberger Rufino ${ }^{1}$ - (D) orcid.org/0000-0003-3799-8313 \\ Alberto Madeiro' \\ Adriana Trinidad ${ }^{1}$ \\ Raiza Santos ${ }^{1}$ \\ Isadora Freitas \\ 'Universidade Estadual do Piauí, Centro de Ciências da Saúde, Teresina-PI, Brasil
}

\section{Resumo}

Objetivo: descrever práticas sexuais e cuidados em saúde de mulheres que fazem sexo com mulheres (MSM). Métodos: estudo transversal, com dados obtidos por questionário eletrônico, de MSM das cinco macrorregiões do país (2013-2014). Resultados: entre 582 MSM, predominaram o sexo oral $(95,2 \%)$ e a penetração vaginal digital $(97,3 \%)$, com raro uso de método de barreira ( $6,7 \%$ e 5,8\%, respectivamente); nos últimos cinco anos, mulheres que fizeram sexo exclusivo com mulher, comparadas àquelas que fizeram sexo com mulher e homem, foram menos propensas a usar método de barreira na prática com mulher $(28,3 \%$ versus $41,1 \% ; \mathrm{p}=0,041)$, realizar consulta anual com ginecologista $(38,9 \%$ vs $70,8 \% ; \mathrm{p}=0,033)$ e receber orientações sobre infecções sexualmente transmissíveis (ISTs) $(44,0 \%$ vs $59,1 \% ; \mathrm{p}=0,034)$ e sobre dúvidas sexuais $(50,0 \%$ vs $63,0 \% ; \mathrm{p}=0,044)$. Conclusão: uso infrequente de métodos de barreira pode ser fator de vulnerabilidade para ISTs; destaca-se a importância da adoção de diretrizes para assistência adequada às MSM.

Palavras-chave: Saúde Sexual; Comportamento Sexual; Homossexualidade Feminina; Saúde Pública; Estudos Transversais.

*Estudo financiado com recursos do Conselho Nacional de Desenvolvimento Científico e Tecnológico (CNPq)/Ministério da Ciência Tecnologia, Inovações e Comunicações (MCTIC): Processo n 472995/2013-8.

Endereço para correspondência:

Andréa Cronemberger Rufino - Rua Olavo Bilac, n² 2335, Centro/Sul, Teresina, PI, Brasil. CEP: 64049-550

E-mail:andreacrufino@gmail.com 


\section{Introdução}

O impacto das sexualidades não hétero na saúde das mulheres está na pauta de pesquisas mundiais desde a década de $19800^{1-3}$ Há uma abundância de dados apontando dificuldades de acesso ${ }^{2-7}$ e experiências negativas durante a assistência médica a mulheres lésbicas e bissexuais, em diversos países. ${ }^{7,8-15}$ Aspectos financeiros, estruturais e culturais nos serviços de saúde foram considerados obstáculos para uma assistência adequada a essas mulheres. ${ }^{2,3} \mathrm{~A}$ utilização menos frequente dos serviços de saúde ${ }^{4,8-11} \mathrm{e}$ os piores indicadores de saúde estão geralmente associados às mulheres lésbicas e bissexuais, comparadas às heterossexuais. $5,6,9,10,16$

\section{Há uma abundância de dados apontando dificuldades de acesso e experiências negativas durante $a$ assistência médica a mulheres lésbicas e bissexuais, em diversos países.}

Critérios distintos têm sido utilizados para caracterizar a lesbianidade e a bissexualidade nos estudos sobre saúde, como práticas sexuais (comportamento sexual), objeto de desejo (orientação sexual) e autoidentidade sexual. ${ }^{2,5} \mathrm{Em}$ algumas pesquisas, a lesbianidade é definida apenas pela prática sexual exclusiva com mulher ou com homem e mulher, resultando na invisibilidade das mulheres bissexuais sob a definição de lésbicas. ${ }^{2} 0$ impacto da lesbianidade e bissexualidade na saúde também pode passar despercebido quando a identidade sexual é o único critério de definição utilizado. ${ }^{4,7}$ Nesse sentido, há sugestões para que as pesquisas sobre lesbianidade e bissexualidade considerem os três critérios supracitados, de maneira a se evitarem vieses na avaliação de indicadores de saúde para esse grupo feminino. ${ }^{2}$

A frequente incoerência entre a identidade sexual autorreferida e as práticas sexuais pode invisibilizar a assistência à saúde das mulheres. ${ }^{4,7} 0$ autocuidado e a busca por assistência médica diferem entre mulheres heterossexuais, lésbicas e bissexuais.7 Há evidências de que exames de prevenção do câncer do colo do útero e de mama são menos realizados por mulheres que se autoidentificam como heterossexuais - mesmo quando essas mesmas mulheres referiam ter práticas sexuais exclusivas com mulher ou, ainda, com mulher e homem -, na comparação com as mulheres autoidentificadas como lésbicas e bissexuais. ${ }^{4}$

As necessidades de saúde sexual e reprodutiva da população lésbica e bissexual feminina apresentam especificidades relacionadas a suas práticas sexuais. ${ }^{910,14,16,17}$ As vulnerabilidades em relação à saúde podem ser mais bem detectadas pelo conhecimento das práticas sexuais, independentemente da sua identidade sexual. Esse argumento justifica a utilização da expressão 'mulheres que fazem sexo com mulheres' (MSM) para agrupar lésbicas e bissexuais em grande número de estudos sobre saúde, lesbianidade e bissexualidade.

A vulnerabilidade conferida pelo exercício de uma sexualidade não hétero está associada à invisibilidade social e, também, a experiências de preconceito nos serviços de saúde. ${ }^{3,7,14,18}$ Há relatos de vivências recorrentes de discriminação e violência contra mulheres lésbicas (lesbofobia) e mulheres bissexuais (bifobia) e, ainda, de invisibilidade, causadas por atitudes heteronormativas dos profissionais de saúde. ${ }^{718} \mathrm{~A}$ heterossexualidade, ao se afirmar como norma social, determina sua existência de forma compulsória e sua superioridade em relação às demais sexualidades. ${ }^{19}$ A imposição de uma heterossexualidade normativa e compulsória é a base de sustentação da lesbofobia e da bifobia. ${ }^{19}$

Experiências negativas durante a assistência médica tornaram as MSM mais resistentes à procura pelos serviços de saúde. ${ }^{7,18}$ Um estudo inglês, publicado em 2011, expôs a atitude dos médicos durante a assistência às mulheres. Os médicos observados naquele estudo demonstraram a crença equivocada de que as mulheres por eles atendidas eram heterossexuais, despertando nelas sentimentos de vergonha, raiva ou desconforto. ${ }^{18}$ A lógica heterossexista, quando valoriza a diferença social entre as mulheres heterossexuais e as não hétero, configura uma das formas mais sutis de lesbofobia e bifobia, ao silenciar e invisibilizar mulheres lésbicas e bissexuais. ${ }^{19}$ Um estudo francês de natureza qualiquantitativa evidenciou o menor acesso das mulheres lésbicas e bissexuais, cujo receio do julgamento médico foi a razão pela qual justificaram a não revelação de suas práticas sexuais durante 0 atendimento de saúde. ${ }^{7}$

No Brasil, há lacunas na produção científica sobre as demandas das MSM relacionadas à saúde sexual e reprodutiva. Não há estudos quantitativos de abrangência nacional sobre tais necessidades, ou que ofertem 
dados sobre a assistência a problemas no exercício da sexualidade. Ademais, desconhece-se a qualidade da assistência ginecológica prestada a essa população em serviços de saúde públicos e privados. Este estudo pretende descrever as práticas sexuais e o cuidados em saúde de mulheres que fazem sexo com mulheres .

\section{Métodos}

Estudo transversal com mulheres que fazem sexo com mulheres das cinco macrorregiões do Brasil, desenvolvido entre julho de 2013 e janeiro de 2014. Um questionário estruturado, anônimo, foi disponibilizado em endereço eletrônico específico, tendo como pré-requisito a concordância com um Termo de Consentimento Livre e Esclarecido (TCLE).

Um inquérito populacional sobre o comportamento sexual dos brasileiros estimou a população de mulheres lésbicas e bissexuais entre 1,7 e 3,0\% das mulheres sexualmente ativas. ${ }^{20}$ No entanto, as condições de vulnerabilidade a que essa população está sujeita, por vivências de estigma e discriminação social, trazem dificuldades para 0 acesso a essas mulheres. ${ }^{20}$ Uma parcela considerável delas não deseja ser reconhecida por suas práticas e identidade sexuais, impondo obstáculos à utilização de uma amostra probabilística e aleatória.

Inicialmente, fez-se contato com um grupo de lésbicas, gays e bissexuais (LGB) da região Nordeste, para a divulgação da pesquisa entre suas representantes. Estas mediaram o contato telefônico e a divulgação do endereço eletrônico do estudo para grupos LGB das demais regiões do país, por redes sociais como Facebook e WhatsApp. Representantes lésbicas e bissexuais de cada região também atuaram como divulgadoras, enviando o convite à participação no estudo para suas redes de contato. Por sua vez, as mulheres que responderam ao instrumento de pesquisa também indicaram outras para participar, pelo mecanismo de 'bola de neve', cuja metodologia tem sido utilizada em estudos sobre sexualidade de MSM. ${ }^{4,21}$ As MSM acessaram o endereço eletrônico que continha o TCLE e 0 instrumento de pesquisa: um questionário eletrônico, a ser enviado quando completamente respondido.

0 instrumento de pesquisa foi construído com perguntas fechadas, distribuídas em três tópicos. 0 primeiro tópico coletou dados sociodemográficos e referentes à autodefinição de práticas e identidade sexuais: faixa etária (em anos: 18-19; 20-29; 30-39; 40-49; $\geq 50$ ), escolaridade (em anos de estudo: $\leq 8$; $9-11 ; \geq 12$ ), região de residência (Norte; Nordeste; Centro-Oeste; Sudeste; Sul), práticas sexuais nos últimos cinco anos (faz sexo com mulher e nunca fez sexo com homem ao longo da vida; faz sexo com mulher e já fez sexo com homem ao longo da vida; faz sexo com mulher e com homem) e identidade sexual (lésbica; bissexual; outra [resposta aberta]). 0 segundo tópico abordou informações sobre tipos de prática sexual e autocuidado em saúde sexual, especialmente no que se refere à adoção de métodos de prevenção de infecções sexualmente transmissíveis (ISTs) e aids: tipo de prática sexual com mulheres (sexo oral; penetração vaginal com dedos; penetração vaginal com brinquedo; penetração anal), tipo de prática sexual com homens (sexo oral; penetração vaginal com dedos; penetração vaginal com pênis; penetração anal) e uso do método de barreira (plástico filme; luva de dedos; preservativos masculino e feminino) na prática sexual com mulher e na prática sexual com homem. 0 terceiro tópico questionou a frequência de procura por atendimento ginecológico nos últimos cinco anos (nenhuma; semestral; anual; sem periodicidade) e as experiências das MSM nos serviços de saúde ginecológicos públicos e privados, relacionadas à atitude das mulheres durante a consulta (revelação sobre orientação sexual; pergunta sobre prevenção de ISTs e aids; pergunta sobre exame citopatológico do colo do útero; pergunta sobre dúvidas sexuais) e à atitude dos médicos (pergunta sobre orientação sexual/práticas sexuais; orientação sobre prevenção de ISTs e aids; orientação sobre exame citopatológico do colo do útero; orientação sobre dúvidas sexuais).

Um projeto-piloto foi desenvolvido com o propósito de testar e aprimorar o instrumento de pesquisa. De início, três pesquisadoras especialistas em estudos de gênero avaliaram as instruções para responder ao questionário, a clareza dos enunciados, a pertinência das perguntas e sua adequação aos objetivos do estudo. Em seguida, o questionário foi disponibilizado a 20 MSM. Nessa etapa, foram analisadas as dificuldades para 0 acesso ao questionário eletrônico e sua utilização, o envio das respostas e a compreensão das perguntas, além do tempo despendido para responder a elas. Após a realização dos ajustes necessários, os dados colhidos no pré-teste foram descartados. 
Durante a pesquisa propriamente dita, as respostas ao questionário foram enviadas a um banco de dados eletrônico e, posteriormente, exportadas para uma planilha eletrônica. Os dados coletados foram analisados por estatística descritiva, mediante a distribuição de frequência dos quesitos 'práticas sexuais', 'aspectos de autocuidado em saúde' e 'experiências das MSM nos serviços de saúde'. Os grupos de mulheres que fazem sexo exclusivamente com mulheres (mulheres que nunca tiveram sexo com homem) e de mulheres que fazem sexo com mulheres e homens (mulheres que já tiveram ou têm sexo com homem) foram comparados, sendo a diferença nas proporções de respostas avaliada pelo teste do qui-quadrado de Pearson. Os dados foram analisados pelo software STATA versão 12.1.

A pesquisa foi aprovada por Comitê de Ética em Pesquisa: Certificado de Apresentação para Apreciação Ética (CAAE) $\mathrm{n}^{0}$ 12710513.9.0000.5209, de 16 de julho de 2013.

\section{Resultados}

Em todo o Brasil, 582 mulheres que fazem sexo com mulheres - de um total de 615 acessos - responderam ao questionário $(94,6 \%)$. Entre as MSM que responderam, $28,4 \%$ eram do Centro-0este, $27,1 \%$ do Sudeste, $22,3 \%$ do Nordeste, $16,5 \%$ do Sul e 5,7\% do Norte do país. A maioria delas pertencia à faixa dos 20 aos 29 anos de idade $(60,3 \%)$ e contavam 12 anos ou mais de estudo (85,2\%). A maior parte, 66,5\%, identificou-se como lésbica, e 31,6\% como bissexual. Em relação aos últimos cinco anos, um terço delas $(34,0 \%)$ informou ter tido práticas sexuais exclusivas com mulher, enquanto cerca da metade $(49,5 \%)$ já teve prática sexual com homem e $16,5 \%$ com mulher e com homem (Tabela 1). A metade das mulheres que se identificaram como lésbicas mencionou ter prática sexual com mulher e já ter tido sexo com homem (dados não apresentados nas tabelas).

Os tipos de práticas sexuais das MSM são mostrados na Tabela 2. As MSM descreveram sexo oral $(95,2 \%)$ e penetração vaginal com dedos $(97,3 \%)$ como práticas frequentes no sexo com mulher. 0 preservativo masculino foi 0 método de barreira mais frequentemente utilizado para a prática de penetração vaginal com brinquedo $(56,5 \%)$ e pe- netração anal $(52,9 \%)$. 0 uso de plástico filme na prática de sexo oral $(6,7 \%)$ e de luva de dedos na penetração vaginal $(5,8 \%)$ ocorreu raramente. No sexo com homem, as MSM informaram que a penetração vaginal com pênis foi a prática mais realizada $(66,1 \%)$, sendo o uso de preservativo masculino mais frequente na penetração vaginal $(87,0 \%)$ e anal $(71,4 \%)$ com pênis.

Houve maior prevalência de consulta anual com ginecologista $(46,7 \%)$ e, preferencialmente, em serviços médicos privados (83,8\%). Durante as consultas, as MSM relataram que 19,0\% dos ginecologistas perguntaram sobre suas práticas sexuais: se com homem, com mulher, ou com mulher e homem. Por sua vez, 58,3\% das MSM revelaram ao ginecologista suas práticas sexuais, fossem elas exclusivas com mulher ou com mulher e homem. As MSM raramente fizeram perguntas ao ginecologista sobre prevenção de ISTs e aids $(14,9 \%)$ ou prevenção do câncer do colo uterino $(10,7 \%)$, ou ainda, sobre suas dúvidas sexuais relacionadas a desejo, excitação e orgasmo (8,5\%). Elas informaram que, quando questionados, pouco mais da metade dos ginecologistas orientou-as a respeito da prevenção de ISTs e aids $(51,4 \%)$, prevenção do câncer do colo uterino $(66,7 \%)$ e dúvidas sexuais $(58,1 \%)$ (Tabela 3).

A Tabela 4 demonstra que, nos últimos cinco anos, as mulheres que fazem sexo apenas com mulher, quando comparadas às mulheres que fazem sexo com mulher e homem, foram menos propensas a usar método de barreira na prática sexual com mulher (28,3\% versus $41,1 \%$; $\mathrm{p}=0,041$ ) e a realizar consulta anual com ginecologista $(38,9 \%$ vs $70,8 \% ; \mathrm{p}=0,033)$. Além disso, quando questionaram os médicos, as mulheres que fazem sexo apenas com mulher receberam menos orientações sobre ISTs e aids $(44,0 \%$ vs $59,1 \%$; $\mathrm{p}=0,034)$ e sobre suas dúvidas sexuais $(50,0 \% v s$ $63,0 \% ; p=0,044)$.

\section{Discussão}

Este artigo relata uma investigação sobre práticas sexuais, cuidados em saúde e experiências durante assistência ginecológica a MSM no Brasil. Os dados coletados evidenciaram que, comparadas às mulheres que fizeram sexo com mulher e homem, mulheres que fizeram sexo exclusivo com mulher foram menos propensas a utilizar método 
Tabela 1 - Características sociodemográficas, práticas e identidades sexuais de mulheres que fazem sexo com mulheres ( $n=582)$, Brasil, 2013-2014

\begin{tabular}{|c|c|c|}
\hline Variáveis & n & $\%$ \\
\hline \multicolumn{3}{|l|}{ Faixa etária (em anos) } \\
\hline $18-19$ & 63 & 10,8 \\
\hline $20-29$ & 351 & 60,3 \\
\hline $30-39$ & 92 & 15,8 \\
\hline $40-49$ & 55 & 9,5 \\
\hline$\geq 50$ & 21 & 3,6 \\
\hline \multicolumn{3}{|l|}{ Escolaridade (em anos de estudo) } \\
\hline Até 8 & 3 & 0,5 \\
\hline $9-11$ & 83 & 14,3 \\
\hline$\geq 12$ & 496 & 85,2 \\
\hline \multicolumn{3}{|l|}{ Região } \\
\hline Norte & 33 & 5,7 \\
\hline Nordeste & 130 & 22,3 \\
\hline Centro-0este & 165 & 28,4 \\
\hline Sudeste & 158 & 27,1 \\
\hline Sul & 96 & 16,5 \\
\hline \multicolumn{3}{|l|}{ Práticas sexuais } \\
\hline Faz sexo com mulher e nunca fez sexo com homem & 198 & 34,0 \\
\hline Faz sexo com mulher e já fez sexo com homem & 288 & 49,5 \\
\hline Faz sexo com mulher e com homem & 96 & 16,5 \\
\hline \multicolumn{3}{|l|}{ Identidade sexual autorreferida } \\
\hline Lésbica & 387 & 66,5 \\
\hline Bissexual & 184 & 31,6 \\
\hline Outra & 11 & 1,9 \\
\hline
\end{tabular}

a) Pansexual/queer/sem rótulos/mulher que faz sexo com mulher/fora do sistema binário/hétero com aventura.

de barreira na prática sexual e a realizar consulta anual com ginecologista; e quando questionaram 0 médico, receberam menos orientações sobre ISTs ou esclarecimentos sobre suas dúvidas sexuais. A decisão de nomear as mulheres segundo suas práticas sexuais não pretendeu ocultar as identidades sexuais autorreferidas ou desconsiderá-las como fator de vulnerabilidade. Ao contrário, a intenção dessa abordagem foi avaliar o comportamento sexual, independentemente de sua identidade, com o objetivo de compreender práticas de cuidado em saúde entre MSM. Pesquisas desenvolvidas em várias partes do mundo evidenciam que $80 \%$ das mulheres lésbicas informaram prática sexual com homem em algum momento de suas trajetórias, uma evidência da fluidez de suas práticas. ${ }^{21-23}$ Esse dado reforça a importância, durante as consultas médicas, da investigação de práticas e identidade sexual no contexto da trajetória de vida das mulheres, para a assistência adequada a suas especificidades de saúde. ${ }^{7}$

Neste estudo, as MSM informaram maior diversidade de práticas sexuais e maior frequência de sexo oral e penetração vaginal digital durante a prática sexual exclusiva com mulher, em comparação às práticas realizadas com homem. Contudo, a utilização de métodos de barreira mostrou-se infrequente, especialmente nas práticas mais comumente relatadas. Mesmo nas práticas penetrativas com brinquedos sexuais, o uso de preservativo masculino foi informado por apenas metade das MSM. A crença arraigada do risco da aquisição de ISTs e aids na prática sexual com homem pode ter influenciado as maiores frequências 
Tabela 2 - Tipo de práticas sexuais e uso do método de barreira entre mulheres que fazem sexo com mulheres, Brasil, 2013-2014

\begin{tabular}{|c|c|c|}
\hline Variáveis & n & $\%$ \\
\hline \multicolumn{3}{|l|}{ Tipo de prática sexual com mulheres ( $n=582$ ) } \\
\hline Sexo oral & 554 & 95,2 \\
\hline Penetração vaginal com dedos & 566 & 97,3 \\
\hline Penetração vaginal com brinquedo & 322 & 55,3 \\
\hline Penetração anal & 157 & 27,0 \\
\hline \multicolumn{3}{|l|}{ Tipo de prática sexual com homens $(n=384)$} \\
\hline Sexo oral & 223 & 58,1 \\
\hline Penetração vaginal com dedos & 201 & 52,3 \\
\hline Penetração vaginal com pênis & 254 & 66,1 \\
\hline Penetração anal & 63 & 16,4 \\
\hline \multicolumn{3}{|l|}{ Método de barreira na prática sexual com mulher } \\
\hline Sexo oral (plástico filme) $(\mathrm{n}=554)$ & 37 & 6,7 \\
\hline Penetração vaginal com dedos (luva de dedos) $(\mathrm{n}=556)$ & 32 & 5,8 \\
\hline Penetração vaginal com brinquedo $(n=322)$ & 182 & 56,5 \\
\hline Penetração anal ( $n=157)$ & 83 & 52,9 \\
\hline \multicolumn{3}{|l|}{ Método de barreira na prática sexual com homem } \\
\hline Sexo oral $(n=223)$ & 65 & 29,1 \\
\hline Penetração vaginal com dedos $(n=201)$ & 10 & 5,0 \\
\hline Penetração vaginal com pênis ( $n=254$ ) & 221 & 87,0 \\
\hline Penetração anal $(n=63)$ & 45 & 71,4 \\
\hline
\end{tabular}

de uso de preservativo masculino pelas MSM em suas práticas sexuais com homem, de acordo com os achados deste estudo. 0 desconhecimento médico sobre práticas sexuais entre mulheres, aliado a uma conduta heteronormativa durante a assistência, torna as MSM invisíveis e destinadas a uma atendimento precário nos serviços de saúde. ${ }^{7,15,17}$

A crença compartilhada por MSM e médicos de que elas não são suscetíveis às ISTs e à aids é recorrente, segundo diversos estudos, favorecendo o desinteresse dessas mulheres na busca por assistência à saúde. ${ }^{7,17}$ Há evidências da prevalência de ISTs e aids em mulheres com prática sexual exclusiva com mulher, embora em percentuais inferiores aos das mulheres com prática sexual com homem..$^{21-26}$ Um estudo publicado em 2013 analisou o uso de métodos de barreira por 1.557 MSM de vários países e mais de $80 \%$ delas relataram nunca ter usado métodos de barreira na prática de sexo oral, com mulher ou com homem. ${ }^{27}$ Semelhantemente aos achados do atual estudo, houve baixa prevalência desse uso durante manipulação genital com dedos
$(11,3 \%)$ e com brinquedos sexuais $(34,4 \%)$, tanto na prática sexual com mulher quanto com homem.

Há dados consistentes a respeito da vulnerabilidade das MSM à infecção pelo papilomavírus humano (HPV) e, por conseguinte, ao câncer do colo uterino. ${ }^{22,26}$ Estudo norte-americano, publicado em 2001, evidenciou a presença de HPV do tipo oncogênico e a prevalência de lesões precursoras do câncer do colo uterino em mulheres com prática sexual exclusiva com mulher ao longo da vida. ${ }^{26}$ Práticas sexuais variadas entre mulheres foram referidas e abrangeram o sexo oral e a manipulação vaginal e anal com dedos e brinquedos sexuais ${ }^{26}$ semelhantes às relatadas pelas MSM brasileiras no presente estudo. Essas evidências reforçam a crença das MSM em serem suscetíveis ao câncer do colo uterino e, portanto, perceberem a necessidade de exames periódicos de prevenção, uma vez que os maiores percentuais de não realização de exame preventivo se referiram a mulheres com identidade lésbica e prática sexual exclusiva com mulher. ${ }^{22,26}$ 
Tabela 3 - Experiências durante assistência ginecológica de mulheres que fazem sexo com mulheres, Brasil, 2013-2014

\begin{tabular}{|c|c|c|}
\hline Variáveis & $\mathbf{n}$ & $\%$ \\
\hline \multicolumn{3}{|l|}{ Consulta com ginecologista nos últimos cinco anos $(n=582)$} \\
\hline Nenhuma & 76 & 13,1 \\
\hline Semestral & 87 & 14,9 \\
\hline Anual & 272 & 46,7 \\
\hline Sem periodicidade & 147 & 25,3 \\
\hline \multicolumn{3}{|l|}{ Cenário das consultas $(n=506)$} \\
\hline Serviço privado & 424 & 83,8 \\
\hline Serviço público & 82 & 16,2 \\
\hline \multicolumn{3}{|l|}{ Atitude das mulheres durante a consulta $(n=506)$} \\
\hline Revelação sobre orientação sexual & 295 & 58,3 \\
\hline Pergunta sobre prevenção de ISTs ${ }^{\mathrm{a}}$ e aids & 74 & 14,6 \\
\hline Pergunta sobre exame citopatológico do colo do útero & 54 & 10,7 \\
\hline Pergunta sobre dúvidas sexuais & 43 & 8,5 \\
\hline \multicolumn{3}{|l|}{ Atitude dos médicos } \\
\hline Pergunta sobre orientação sexual/práticas sexuais $(n=506)$ & 96 & 19,0 \\
\hline Orientação sobre prevenção de ISTs ${ }^{\mathrm{a}} \mathrm{e}$ aids ( $\mathrm{n}=74$ ) & 38 & 51,4 \\
\hline Orientação sobre exame citopatológico do colo do útero $(n=54)$ & 36 & 66,7 \\
\hline Orientação sobre dúvidas sexuais $(n=43)$ & 25 & 58,1 \\
\hline
\end{tabular}

a) ISTs: infecçōes sexualmente transmissiveis.

Tabela 4 - Práticas sexuais e experiências relatadas durante consulta médica entre mulheres que fazem sexo apenas com mulheres e mulheres que fazem sexo com mulheres e homens, Brasil, 2013-2014

\begin{tabular}{lccc}
\hline Variáveis & $\begin{array}{c}\text { MSME } \\
(\mathbf{n}=198)\end{array}$ & $\begin{array}{c}\text { MSMH } \\
(\mathbf{n}=384)\end{array}$ & Valor de $\mathbf{p}^{\mathrm{c}}$ \\
\cline { 1 - 3 } Método de barreira no sexo oral com mulher & $\%$ & $\%$ & 0,437 \\
Método de barreira no sexo vaginal com mulher & 6,1 & 6,5 & 0,041 \\
Método de barreira no sexo anal com mulher & 28,3 & 41,1 & 0,451 \\
Consulta anual com ginecologista & 53,5 & 52,6 & 0,033 \\
Revelação sobre prática sexual com mulher & 38,9 & 70,8 & 0,323 \\
Pergunta sobre ISTs ${ }^{\mathrm{d}}$ e aids & 63,5 & 57,2 & 0,364 \\
Pergunta sobre exame citopatológico do colo do útero & 15,7 & 14,5 & 0,763 \\
Pergunta sobre dúvidas sexuais & 10,7 & 10,9 & 0,512 \\
Médico perguntou sobre prática sexual com mulher & 10,1 & 8,0 & 0,215 \\
Orientação sobre ISTs ${ }^{\mathrm{d}}$ e aids & 20,1 & 18,9 & 0,034 \\
Orientação sobre exame citopatológico do colo do útero & 44,0 & 59,1 & 0,344 \\
Orientação sobre dúvidas sexuais & 64,7 & 67,7 & 0,044 \\
\hline
\end{tabular}

a) Mulheres que fizeram sexo com mulheres exclusivamente.

b) Mulheres que fizeram sexo com mulheres e homens.

c) Teste do qui-quadrado de Pearson.

d) ISTs: infecções sexualmente transmissíveis. 
As dificuldades para o acesso das MSM aos serviços de saúde incluem barreiras estruturais, financeiras e culturais. A atitude heteronormativa dos profissionais foi apontada como o maior obstáculo a uma adequada assistência à saúde. ${ }^{2,3,15}$ As dificuldades para revelar as práticas sexuais ${ }^{5,6,14,15}$ e as experiências anteriores de discriminação, mesmo depois dessa revelação, constituem relatos recorrentes de MSM em estudos realizados mundo afora. ${ }^{7,14,17,21}$ Nesta investigação em particular, quase metade das MSM buscaram assistência ginecológica anual em serviços predominantemente privados. A alta escolaridade das MSM pode ter influenciado na escolha desses serviços, como aponta a Pesquisa Nacional de Saúde de $2013{ }^{28}$ Porém, essa procura foi significativamente menor entre aquelas que relataram práticas sexuais de forma exclusiva com mulher nos últimos cinco anos. Apenas uma minoria de ginecologistas perguntou às MSM sobre práticas sexuais com mulher, com homem ou com ambos os sexos. A heterossexualidade normativa, dominante no discurso médico, impõe dificuldades ao diálogo e à assistência, no que se refere às individualidades das MSM., ${ }^{713-15,17}$

A decisão de revelar práticas e identidade sexual é relatada pelas MSM como um momento de tensão e ansiedade, acentuado pelo medo de vivenciar discriminação e preconceito. ${ }^{4,14}$ A trajetória de vida marcada por experiências de lesbofobia e bifobia, compartilhada por muitas MSM, pode interferir negativamente nessa decisão ${ }^{4-6,14}$ As experiências de 121 mulheres lésbicas e bissexuais nos serviços de saúde da Noruega foi objeto de uma pesquisa publicada em 2009, e segundo os relatos dessas mulheres, a maioria dos médicos e psicólogos não lhes perguntaram, tampouco facilitaram a revelação de sua lesbianidade ou bissexualidade; a qualidade da assistência a elas prestada foi prejudicada por atitudes heteronormativas e pela falta de conhecimento dos profissionais sobre as sexualidades não hétero e suas necessidades singulares de saúde. ${ }^{15}$

De volta ao presente estudo, $88 \%$ das MSM não perguntaram aos ginecologistas sobre prevenção de ISTs e câncer do colo uterino, tampouco apresentaram a eles suas dúvidas de ordem sexual. Quando questionaram os ginecologistas, três em cada cinco MSM receberam orientações, porém com menor propensão de resposta para as mulheres que relataram sexo apenas com mulher nos últimos cinco anos, principalmente ao exporem dúvidas e queixas de ordem sexual ou solicitarem esclarecimento sobre prevenção de ISTs. Esse dado pode refletir o desconhecimento dos médicos a respeito das práticas sexuais entre mulheres e, consequentemente, de como orientá-las na prevenção de ISTs. A crença na heterossexualidade compulsória das mulheres pode prejudicar a escuta, atrasar diagnósticos e resultar na oferta de tratamentos indevidos., ${ }^{74,17,21}$ Há relatos de atitudes inadequadas dos profissionais, quando confrontados com informações ou dúvidas sobre práticas sexuais com mulher ou com mulher e homem. Na percepção das MSM, os médicos agem de forma inadequada diante dessas situações, com prejuízo na identificação de suas demandas específicas de saúde. ${ }^{7,13,14,17}$

A decisão de utilizar um método exploratório com uma amostra não probabilística impôs limitações ao estudo. 0 perfil da amostra de conveniência composta por MSM conectadas à comunidade LGB pode não ser representativo das MSM brasileiras, impedindo a generalização dos resultados. Entretanto, a escolha de uma amostra de conveniência é justificável, haja vista as dificuldades de acesso a essa população de mulheres, conforme relatos de diversas pesquisas realizadas em outros países. ${ }^{21,25-27}$ Esse tipo de amostra também pode ter limitado 0 acesso a mulheres de faixa etária mais elevada, de menor escolaridade e residentes em regiões com dificuldades de acesso à internet: na região Norte, por exemplo, houve maior obstáculo ao contato com as MSM, resultando em menor eficácia do mecanismo de 'bola de neve' e, como consequência, menor número de participantes no estudo. Porém, o uso da internet permitiu uma proporção elevada de resposta, aliada a uma redução de custos operacionais, considerando-se a extensão do país. A despeito das limitações descritas, trata-se do primeiro estudo quantitativo de abrangência nacional sobre práticas sexuais, cuidados em saúde e assistência ginecológica de MSM no Brasil.

A atitude das MSM em revelar suas práticas e identidade sexuais pode significar uma tentativa de romper com um ciclo de vulnerabilidades imposto pela heteronorma. É provável que a participação em grupos de militância LGB empodere as MSM, no sentido de uma autoestima positiva da identidade lésbica e bissexual. Já a não revelação dessa identidade por parte de quase metade das MSM, possivelmente, refletiria experiências anteriores de discriminação e preconceito em diversos contextos; ao mesmo tempo, outros marcadores de vulnerabilidade não pesquisados teriam contribuído para essa limitação, como idade, raça/etnia e condição 
socioeconômica. Estudos de caráter etnográfico com base em entrevistas semiestruturadas podem conduzir a um melhor esclarecimento dessa questão. Diante deste panorama, a atitude proativa dos profissionais de saúde em questionar sobre práticas e identidade sexual pode resultar no reconhecimento das vulnerabilidades, no acolhimento e na humanização da assistência a essas mulheres.

A diversidade de práticas sexuais informadas pelas MSM, associada ao uso infrequente de métodos de barreira, torna-as mais suscetíveis a ISTs e aids. 0 conhecimento médico das práticas sexuais, independentemente da identidade sexual autorreferida pelas MSM, pode influenciar positivamente na identificação de sua suscetibilidade ao câncer do colo uterino, ISTs e aids. Por sua vez, o conhecimento da identidade sexual das MSM permite a identificação de suas vulnerabilidades e de como estas intensificam o impacto negativo sobre a saúde. Para o profissional, são conhecimentos que favorecem a oferta de assistência adequada às necessidades singulares de saúde dessa população de mulheres.

A elevada procura por consultas ginecológicas pode significar uma atitude de autocuidado das MSM com a saúde, assim como a busca por assistência ginecológica ser resultado de necessidades de saúde específicas dessa especialidade médica não supridas por outros profissionais. Uma minoria de MSM perguntou aos

\section{Referências}

1. Boehmer U. Twenty years of public health research: inclusion of lesbian, gay, bisexual and transgender populations. Am J Public Health. 2002 Jul;92(7):1125-30.

2. Institute of Medicine (US) Committee on Lesbian Health Research Priorities; Solarz AL. Lesbian health: current assessment and directions for the future [Internet]. Washington, DC: National Academy Press; 1999 [cited 2017 Feb 15]. 256 p. Available in: http://www.ncbi.nlm. nih.gov/books/NBK45100/?report=reader

3. Gay and lesbian medical association; LGBT Health Experts. Healthy people 2010: companion document for lesbian, gay, bisexual and transgender (LGBT) health [Internet]. San Francisco, LA: Gay and Lesbian Medical Association; 2001 [cited 2017 Feb 15]. 481 p. Available in: https://www.med.umich.edu/diversity/ pdffiles/healthpeople.pdf

4. Kerker BD, Mostashari F, Thorpe L. Health care access and utilization among women who have sex with ginecologistas sobre temas afins à saúde sexual e reprodutiva. Mesmo diante desse interesse, as perguntas foram respondidas por apenas metade dos profissionais com quem se consultaram. A assistência ginecológica recebida pelas MSM expõe a precariedade do reconhecimento e da atenção prestada a sua orientação sexual pelos serviços de saúde brasileiros. É necessário ressaltar a importância da visibilidade das MSM nas políticas públicas de atenção à saúde. A formação médica voltada para as singularidades de saúde das MSM e a definição de diretrizes para a abordagem de tais especificidades são mandatórias, especialmente quanto à infecção pelo HPV, outras ISTs e aids. 0 impacto negativo da invisibilidade ainda pode resultar na perda de uma janela de oportunidades para prevenção, diagnóstico e tratamento de diversas doenças.

\section{Contribuição dos autores}

Rufino AC e Madeiro A contribuíram na concepção do estudo, análise e interpretação dos dados e redação do manuscrito. Trinidad A, Santos R e Freitas I participaram do levantamento e análise de dados e da revisão crítica do conteúdo intelectual do artigo. Todos os autores aprovaram a versão final e se responsabilizam por todos os aspectos do trabalho, garantindo sua precisão e integridade.

women: sexual behavior and identity. J Urban Health. 2006 Sep;83(5):970-9.

5. Tjepkema M. Health care use among gay, lesbian and bisexual Canadians. Health Rep. 2008 Mar;19(1):53-64.

6. Fredriksen-Goldsen KI, Kim HJ, Barkan SE, Muraco A, Hoy-Ellis CP. Health disparities among lesbian, gay, and bisexual older adults: results from a population based study. Am J Public Health. 2013 Oct;103(10):1802-9.

7. Chetcuti N, Beltzer N, Methy N, Laborde C, Velter A, Bajos N, et al. Preventive care's forgotten women: life course, sexuality, and sexual health among homosexually and bisexually active women in France. J Sex Res. 2013;50(6):587-97.

8. Dilley JA, Simmons KW, Boysun MJ, Pizacani BA, Stark MJ. Demonstrating the importance and feasibility of including sexual orientation in public health surveys: health disparities in the Pacific Northwest. Am J Public Health. 2010 Mar;100(3):460-7. 
9. Ward BW, Dahlhamer JM, Galinsky AM, Joestl SS. Sexual orientation and health among U.S. adults: national health interview survey, 2013. Natl Health Stat Report. 2014 Jul;77:1-10.

10. King M, Semlyen J, Tai SS, Killaspy H, Osborn D, Popelyuk D, et al. A systematic review of mental disorder, suicide, and deliberate self harm in lesbian, gay, and bisexual people. BMC Psychiatry. 2008;8:70.

11. King M, Nazareth I. The health of people classified as lesbian, gay, and bisexual attending family practitioners in London: a controlled study. BMC Public Health. 2006 May;6:127.

12. Davy Z, Siriwardena AN. To be or not to be LGBT in primary health care: heath care for lesbian, gay, bisexual, and transgender people. Br J Gen Pract. 2012 Sep;62(602):491-2.

13. Meads C, Buckley E, Sanderson P. Ten years of lesbian health survey research in the UK West Midlands. BMC Public Health. 2007 Sep; 7:251.

14. Bjorkman M, Malterud K. Lesbian women's experiences with health care: a qualitative study. Scand J Prim Health Care. 2009;27(4):238-43.

15. McNair R. Lesbian and bisexual women's sexual health. Aust Fam Physician. 2009 Jun;38(6):388-93.

16. McLaughlin KA, Hatzenbuehler ML, Keyes KM. Responses to discrimination and psychiatric disorders among Black, hispanic, female, and lesbian, gay, and bisexual individuals. Am J Public Health. 2010 Aug;100(8):1477-84.

17. Barbosa RM, Fachini R. Acesso a cuidados relativos à saúde sexual entre mulheres que fazem sexo com mulheres em São Paulo, Brasil. Cad Saúde Pública. 2009;25(suppl 2):S291-300.

18. Formby E. Lesbian and bisexual women's human rights, sexual rights and sexual citizenship: negotiating sexual health in England. Cult Health Sex. 2011 Nov;13(10):1165-79.

19. Butler J. Regulaciones de género. Rev Est Género La Ventana. 2006;23:7-35.
20. Barbosa RM, Koyama MAH. Mulheres que fazem sexo com mulheres: algumas estimativas para o Brasil. Cad Saúde Pública. 2006 Jul;22(7):1511-14.

21. Pinto VM, Tancredi MV, Tancredi Neto A, Buchalla CM. Sexually transmitted disease/HIV risk behavior among women who have sex with women. AIDS. 2005 Oct;19(suppl 4):\$64-9.

22. Henderson HJ. Why lesbians should be encouraged to have regular cervical screening. J Fam Plann Reprod Health Care. 2009 Jan;35(1):49-52.

23. Gorgos LM, Marrazzo JM. Sexually transmitted infections among women who have sex with women. Clin Infect Dis. 2011 Dec;53(suppl 3):584-91.

24. Chan SK, Thornton LR, Chronister KJ, Meyer J, Wolverton M, Johnson CK, et al. Likely femaleto-female sexual transmission of HIV - Texas, 2012. MMWR Morb Mortal Wkly Rep. 2014 Mar;63(10):209-12.

25. Wang XF, Norris JL, Liu YJ, Reilly KH, Wang N. Health-related attitudes and risk factors for sexually transmitted infections of Chinese women who have sex with women. Chin Med J (Engl). 2012 Aug;125(16):2819-25.

26. Marrazzo JM, Koutsky LA, Kiviat NB, Kuypers JM, Stine K. Papanicolaou test screening and prevalence of genital human papillomavirus among women who have sex with women. Am J Public Health. 2001 Jun;91(6):947-52.

27. Rowen TS, Breyer BN, Lin TC, Li CS, Robertson PA, Shindel AW. Use of barrier protection for sexual activity among women who have sex with women. Int J Gynaecol Obstet. 2013 Jan;120(1):42-5.

28. Ministério da Saúde (BR); Ministério do Planejamento, Orçamento e Gestão (BR); Instituto Brasileiro de Geografia e Estatística. Pesquisa nacional de saúde 2013. Percepção do estado de saúde, estilos de vida e doenças crônicas [Internet]. Rio de Janeiro: Instituto Brasileiro de Geografia e Estatística; 2014 [citado 2018 mar 10]. 181 p. Disponível em: ftp://ftp.ibge.gov.br/PNS/2013/ pns2013.pdf 


\begin{abstract}
Objective: to describe sexual practices and health care of women who have sex with women (WSW). Methods: this was a cross-sectional study with data obtained by means of an electronic questionnaire answered by WSW from the five regions of the country (2013-2014). Results: among 582 WSW, oral sex (95.2\%) and digital penetration of the vagina (97.3\%) were predominant, with rare use of barrier methods $(6.7 \%$ and $5.8 \%$, respectively); in the last five years, women who had sex exclusively with women, compared to those who had sex with women and men, were less likely to use barrier method with women (28.3\% versus $41.1 \%$; $p=0.041)$, less likely to have an annual check-up with a gynecologist (38.9\% vs 70.8\%; $p=0.033$ ), less likely to receive guidance about sexually transmitted infections (STIS) $(44.0 \%$ vs $59.1 \% ; p=0.034)$ and about sexual doubts $(50.0 \%$ vs $63.0 \%$; $p=0.044)$. Conclusion: infrequent use of barrier methods may be a vulnerability factor for STIs; it highlights the importance of guidelines for adequate bealth care for WSW.
\end{abstract}

Keywords: SexualHealth; SexualBehavior; Homosexuality, Female; Public Health; Cross-Sectional Studies.

\section{Resumen}

Objetivo: describir prácticas sexuales y cuidados en salud de mujeres que tienen sexo con mujeres (MSM). Métodos: estudio transversal con datos obtenidos por cuestionario electrónico, de MSM de las cinco grandes regiones del país (2013-2014). Resultados: entre 582 MSM, predominaron el sexo oral (95,2\%) y la penetración vaginal digital (97,3\%), con poco uso de método de barrera $(6,7 \%$ y $5,8 \%$, respectivamente); en los últimos cinco años, mujeres que tuvieron sexo exclusivo con mujer, comparadas a las que tuvieron sexo con mujer y hombre, fueron menos propensas a usar método de barrera con mujer (28,3\% versus 41,1\%; $p=0,041)$, a realizar consulta anual con ginecólogo (38,9\% vs 70,8\%; $p=0,033)$ y a recibir orientaciones sobre infecciones de transmisión sexual (ITS) (44,0\% vs 59,1\%; $p=0,034)$ y sobre dudas sexuales (50,0\% vs 63,0\%; $p=0,044)$. Conclusión: el uso infrecuente de métodos de barrera puede ser factor de vulnerabilidad para ITS; se destaca la importancia de la adopción de directrices para asistencia adecuada de MSM.

Palabras clave: Salud Sexual; Conducta Sexual; Homosexualidad Femenina; Salud Pública; Estudios Transversales.

Recebido em 07/01/2018

Aprovado em 25/07/2018 